\title{
Choquet integrals, weighted Hausdorff content and maximal operators
}

\section{Lin Tang}

\begin{abstract}
The boundedness of maximal operators on the weighted Choquet space and the Choquet-Morrey space is established. These results are used to study Carleson embeddings for weighted Sobolev spaces.
\end{abstract}

Keywords. Choquet integral, maximal operator, Carleson embedding.

2010 Mathematics Subject Classification. 42B25, 28A12.

\section{Introduction}

The purpose of this note is to establish the boundedness of maximal operators on the weighted Choquet space and the Choquet-Morrey space. Let us first introduce some notation.

The Choquet integral of $\phi \geq 0$ with respect to a set function $\mathscr{L}$ is defined by

$$
\int \phi d \mathscr{L}=\int_{0}^{\infty} \mathscr{L}\left\{x \in \mathbb{R}^{n}: \phi>t\right\} d t .
$$

Let $0 \leq \beta<n$; the maximal function of $f$ of order $\beta$ is defined by

$$
M_{\beta} f(x)=\sup _{x \in Q}|Q|^{\frac{\beta}{n}-1} \int_{Q}|f(y)| d y,
$$

where the supremum is taken over all cubes $Q$ with sides parallel to the coordinate axes and $|Q|$ denotes the $n$-dimensional volume of $Q$. For convenience, $M_{0}$ is replaced by $M$, that is, $M$ is the Hardy-Littlewood maximal operator.

If $E \subset \mathbb{R}^{n}, \omega \in A_{1}\left(\mathbb{R}^{n}\right)$ and $0<\alpha \leq n$, then the $\alpha$-dimensional weighted Hausdorff content of $E$ (see [7]) is defined by

$$
H_{\omega}^{\alpha}(E)=\inf \sum_{j=1}^{\infty} \frac{\omega\left(Q_{j}\right)}{\left|Q_{j}\right|} l\left(Q_{j}\right)^{\alpha},
$$


where the infimum is taken over all coverings of $E$ by countable families of cubes $Q_{j}$ with sides parallel to the coordinate axes. Here $l(Q)$ denotes the side length of the cube $Q$ and $\omega(Q)=\int_{Q} w(x) d x$. The Muckenhoupt class $A_{1}\left(\mathbb{R}^{n}\right)$ is defined by

$$
M(\omega)(x) \leq C \omega(x), \quad \text { a.e. } x \in \mathbb{R}^{n} .
$$

Remark 1. If we take the infimum in (2) only over coverings of $E$ by dyadic cubes, we obtain an equivalent quantity $H_{w, d}^{\alpha}$ called the dyadic $\alpha$-dimensional weighted Hausdorff content; see Proposition 3.4.2 in [7].

Now we can state our main results.

Theorem 1. Let $\omega \in A_{1}\left(\mathbb{R}^{n}\right), 0 \leq \beta<n, 0<\alpha<n$ and $0<\alpha+\beta<n$. If $\alpha /(n-\beta)<p<(n-\alpha) / \beta$, then

$$
\int_{\mathbb{R}^{n}}\left(M_{\beta} f\right)^{p} d H_{\omega}^{\alpha} \leq C \int_{\mathbb{R}^{n}}|f|^{p} d H_{\omega}^{\alpha+p \beta},
$$

where $C$ depends only on $\alpha, \beta, n, p$ and the constant $A_{1}\left(\mathbb{R}^{n}\right)$.

Let $0 \leq \lambda, 0<p$ and $0<\alpha \leq n$. We define the Choquet-Morrey space $H_{p}^{\alpha, \lambda}$ by

$$
\|f\|_{H_{p}^{\alpha, \lambda}}:=\sup _{x_{0} \in \mathbb{R}^{n}, r>0} r^{-\lambda} \int_{0}^{\infty} H^{\alpha}\left(\left\{y \in B\left(x_{0}, r\right):|f(y)|^{p}>t\right\}\right) d t<\infty .
$$

Theorem 2. Let $0 \leq \beta<n, 0<\alpha<n$ and $0<\alpha+\beta<n$. If $\alpha /(n-\beta)<p<$ $(n-\alpha) / \beta$ and $0 \leq \lambda<\alpha$, then

$$
\left\|M_{\beta} f\right\|_{H_{p}^{\alpha, \lambda}} \leq C\|f\|_{H_{p}^{\alpha+p \beta, \lambda}},
$$

where $C$ depends only on $\alpha, \beta, n, p$ and $\lambda$. Let $p=\alpha /(n-\beta)$. Then

$$
H^{\alpha}\left(\left\{y \in B(x, r):\left|M_{\beta} f(y)\right|>t\right\}\right) \leq C t^{-\alpha /(n-\beta)} r^{\lambda}\|f\|_{H_{\frac{\alpha}{n-\beta}}^{\frac{n \alpha}{n-\beta}, \lambda}},
$$

where $C$ depends only on $\alpha, \beta, n$ and $\lambda$.

Obviously, our results extend some well-known results for maximal operators; see [2] and [6]. 


\section{Proof of the main theorems}

Proof of Theorem 1. We follow the same scheme as in [6]. Without loss of generality, we can assume that $f \geq 0$.

Obviously, to prove Theorem 1, we only need to consider the dyadic case by Remark 1. By the definition of $H_{\omega, d}^{\alpha}$, for each integer $k$, we can take a family of non-overlapping dyadic cubes $Q_{j}^{(k)}$ such that

$$
\left\{x: 2^{k}<f(x) \leq 2^{k+1}\right\} \subset \bigcup_{j} Q_{j}^{(k)}
$$

and

$$
\sum_{j} \frac{\omega\left(Q_{j}^{(k)}\right)}{\left|Q_{j}^{(k)}\right|} l\left(Q_{j}^{(k)}\right)^{\alpha+p \beta} \leq 2 H_{\omega, d}^{\alpha+p \beta}\left(\left\{x: 2^{k}<f \leq 2^{k+1}\right\}\right) .
$$

Set $g=\sum_{k} 2^{(k+1) p} \chi_{A_{k}}$, where $A_{k}=\bigcup_{j} Q_{j}^{(k)}$ and $\chi_{E}$ is the characteristic function of the set $E$. Thus $f^{p} \leq g$.

Let $0 \leq \gamma<n$ and $\alpha /(n-\gamma)<q$. We first claim that for any cube $Q$,

$$
\int M_{\gamma}\left(\chi_{Q}\right)^{q} d H_{\omega}^{\alpha} \leq C \frac{\omega(Q)}{|Q|} l(Q)^{\alpha+q \gamma} .
$$

Indeed, let $x_{Q}$ be the center of $Q$. Then

$$
M_{\gamma}\left(\chi_{Q}\right)(x) \leq C_{0} \frac{l(Q)^{n}}{\left(l(Q)+\left|x-x_{Q}\right|\right)^{n-\gamma}}, \quad x \in \mathbb{R}^{n} .
$$

By the property of $A_{1}\left(\mathbb{R}^{n}\right)$ and the definition of $H_{\omega}^{\alpha}$, we then get

$$
\begin{aligned}
\int_{0}^{\infty} & H_{\omega}^{\alpha}\left(\left\{y:\left|M_{\gamma} \chi_{Q}(y)\right|^{q}>t\right\}\right) d t \\
& \leq \int_{0}^{\infty} H_{\omega}^{\alpha}\left(\left\{y: C_{0} \frac{l(Q)^{n}}{\left(l(Q)+\left|y-x_{Q}\right|\right)^{n-\gamma}}>t^{1 / q}\right\}\right) d t \\
& \leq \int_{0}^{\left(C_{0} l(Q)^{\gamma}\right)^{q}} H_{\omega}^{\alpha}\left(\left\{y: C_{0} \frac{l(Q)^{n}}{\left|y-x_{Q}\right|^{n-\gamma}}>t^{1 / q}\right\}\right) d t \\
& =\int_{0}^{\left(C_{0} l(Q)^{\gamma}\right)^{q}} H_{\omega}^{\alpha}\left(\left\{y:\left|y-x_{Q}\right|<\left(\frac{C_{0} l(Q)^{n}}{t^{1 / q}}\right)^{\frac{1}{n-\gamma}}\right\}\right) d t \\
& \leq \int_{0}^{\left(C_{0} l(Q)^{\gamma}\right)^{q}} \frac{\omega\left(B\left(x_{Q},\left(\frac{C_{0} l(Q)^{n}}{t^{1 / q}}\right)^{\frac{1}{n-\gamma}}\right)\right)}{\left|B\left(x_{Q},\left(\frac{C_{0} l(Q)^{n}}{t^{1 / q}}\right)^{\frac{1}{n-\gamma}}\right)\right|}\left(\frac{C_{0} l(Q)^{n}}{t^{1 / q}}\right)^{\frac{\alpha}{n-\gamma}} d t
\end{aligned}
$$




$$
\begin{aligned}
& \leq C \frac{\omega(Q)}{|Q|} \int_{0}^{\left(C_{0} l(Q)^{\gamma}\right)^{q}}\left(\frac{C_{0} l(Q)^{n}}{t^{1 / q}}\right)^{\frac{\alpha}{n-\gamma}} d t \\
& \leq C \frac{\omega(Q)}{|Q|} l(Q)^{\alpha+q \gamma} .
\end{aligned}
$$

The last inequality holds since $\alpha /(n-\gamma)<q$. Thus (3) is proved.

Let us proceed to the proof. Assume first that $1 \leq p<(n-\alpha) / \beta$. Then

$$
\left(M_{\beta} f\right)^{p} \leq M_{p \beta}\left(f^{p}\right) \leq M_{p \beta}(g) \leq \sum_{k} 2^{(k+1) p} \sum_{j} M_{p \beta}\left(\chi_{Q_{j}^{(k)}}\right) .
$$

Taking $q=1$ and $\gamma=p \beta$ in (3) and using the subadditivity of weighted Choquet integrals, we have

$$
\begin{aligned}
\int\left(M_{\beta} f\right)^{p} d H_{\omega, d}^{\alpha} & \leq C \sum_{k} 2^{(k+1) p} \sum_{j} \int M_{p \beta}\left(\chi_{Q_{j}^{(k)}}\right) d H_{\omega, d}^{\alpha} \\
& \leq C \sum_{k} 2^{(k+1) p} \frac{\omega\left(Q_{j}^{(k)}\right)}{\left|Q_{j}^{(k)}\right|} l\left(Q_{j}^{(k)}\right)^{\alpha+p \beta} \\
& \leq C \sum_{k} 2^{(k+1) p} H_{\omega, d}^{\alpha+p \beta}\left(\left\{x: 2^{k}<f(x) \leq 2^{k+1}\right\}\right) \\
& \leq C \sum_{k} \frac{2^{2 p}}{2^{p}-1} \int_{2^{(k-1) p}}^{2^{k p}} H_{\omega, d}^{\alpha+p \beta}\left(\left\{x: f(x)^{p}>t\right\}\right) d t \\
& \leq C \int f^{p} d H_{\omega, d}^{\alpha+p \beta} .
\end{aligned}
$$

Assume now that $\alpha /(n-\beta)<p<1$. Note that $f \leq \sum_{k} 2^{k+1} \chi_{A_{k}}$,

$$
M_{\beta} f \leq \sum_{k} 2^{k+1} \sum_{j} M_{\beta}\left(\chi_{Q_{j}^{(k)}}\right) .
$$

Taking $q=p$ and $\gamma=\beta$ in (3), we obtain

$$
\int\left(M_{\beta}\right)^{p} d H_{\omega, d}^{\alpha} \leq C \sum_{k} 2^{(k+1) p} \frac{\omega\left(Q_{j}^{(k)}\right)}{\left|Q_{j}^{(k)}\right|} l\left(Q_{j}^{(k)}\right)^{\alpha+p \beta} \leq C \int f^{p} d H_{\omega, d}^{\alpha+p \beta} .
$$

The proof is complete.

Let us now turn to the proof of Theorem 2 . 
Proof of Theorem 2. Without loss of generality, we can assume $\lambda>0$. Choose any $x_{0} \in \mathbb{R}^{n}$ and $r>0$, and write

$$
f(x)=f_{0}(x)+\tilde{f}_{0}(x),
$$

where $f_{0}=\chi_{B\left(x_{0}, 2 r\right)}$.

For the term $f_{0}$, from Theorem 1, we have

$$
\begin{aligned}
\int_{0}^{\infty} H^{\alpha}\left(\left\{y \in B\left(x_{0}, r\right)\right.\right. & \left.\left.:\left|M_{\beta} f_{0}(y)\right|^{p}>t\right\}\right) d t \\
\leq & C \int_{0}^{\infty} H^{\alpha+p \beta}\left(\left\{y \in B\left(x_{0}, 2 r\right):|f(y)|^{p}>t\right\}\right) d t .
\end{aligned}
$$

Hence

$$
\left\|M_{\beta} f_{0}\right\|_{H_{p}^{\alpha, \lambda}} \leq C\|f\|_{H_{p}^{\alpha+p \beta, \lambda} .}
$$

Now consider the term $\tilde{f}_{0}$. Let $q=p n /(\alpha+p \beta)>1$. Then for any $y \in B\left(x_{0}, r\right)$, we have

$$
\begin{aligned}
M_{\beta}\left(\tilde{f}_{0}\right)(y) & \leq C \sum_{k=1}^{\infty}\left(2^{k} r\right)^{\beta-n} \int_{B\left(x_{0}, 2^{k+1} r\right)}|f(x)| d x \\
& \leq C \sum_{k=1}^{\infty}\left(2^{k} r\right)^{\beta-n / q}\left(\int_{B\left(x_{0}, 2^{k+1} r\right)}|f(x)|^{q} d x\right)^{1 / q} \\
& \leq C \sum_{k=1}^{\infty}\left(2^{k} r\right)^{\beta-n / q}\left(\int_{B\left(x_{0}, 2^{k+1} r\right)}|f(x)|^{p} d H^{\alpha+p \beta}\right)^{1 / p} \\
& \leq C \sum_{k=1}^{\infty}\left(2^{k} r\right)^{(\lambda-\alpha) / p}\|f\|_{H_{p}^{\alpha+p \beta, \lambda}}^{1 / p} \\
& \leq C r^{(\lambda-\alpha) / p}\|f\|_{H_{p}^{\alpha+p \beta, \lambda}}^{1 / p},
\end{aligned}
$$

since $\lambda<\alpha$ and in the third inequality we use the inequality (see [6])

$$
\int f(x) d x \leq \frac{n}{\alpha}\left(\int f^{\alpha / n} d H^{\alpha}\right)^{n / \alpha}
$$

for $0<\alpha \leq n$.

Thus

$$
\begin{aligned}
\int_{0}^{\infty} & H^{\alpha}\left(\left\{y \in B\left(x_{0}, r\right):\left|M_{\beta}\left(\tilde{f}_{0}\right)(y)\right|^{p}>t\right\}\right) d t \\
& \leq \int_{0}^{\infty} H^{\alpha}\left(\left\{y \in B\left(x_{0}, r\right): C r^{(\lambda-\alpha)}\|f\|_{\left.\left.H_{p}^{\alpha+p \beta, \lambda}>t\right\}\right) d t}\right.\right.
\end{aligned}
$$




$$
\begin{aligned}
& \leq \int_{0}^{C r^{(\lambda-\alpha)}\|f\|_{H_{p}^{\alpha+p \beta, \lambda}}} H^{\alpha}\left(B\left(x_{0}, r\right)\right) d t \\
& \leq C r^{\lambda}\|f\|_{H_{p}^{\alpha+p \beta, \lambda} .}
\end{aligned}
$$

Therefore

$$
\left\|M_{\beta} \tilde{f}_{0}\right\|_{H_{p}^{\alpha, \lambda}} \leq C\|f\|_{H_{p}^{\alpha+p \beta, \lambda}}
$$

From these inequalities we obtain

$$
\left\|M_{\beta} f\right\|_{H_{p}^{\alpha, \lambda}} \leq C\left(\left\|M_{\beta} f_{0}\right\|_{H_{p}^{\alpha, \lambda}}+\left\|M_{\beta} \tilde{f}_{0}\right\|_{H_{p}^{\alpha, \lambda}}\right) \leq C\|f\|_{H_{p}^{\alpha+p \beta, \lambda}} .
$$

As for the case $p=\alpha /(n-\beta)$, the proof is the same replacing Theorem 1 by the corresponding weak estimate, that is, for any $t>0$

$$
H^{\alpha}\left(\left\{y:\left|M_{\beta} f(y)\right|>t\right\}\right) \leq C t^{-\alpha /(n-\beta)} \int f^{\frac{\alpha}{n-\beta}} d H^{\frac{n \alpha}{n-\beta}} .
$$

The proof of (4) can be found in [6].

\section{An application}

In this section, we study the Carleson embeddings for weighted Sobolev spaces.

To state our result, let us introduce some notation.

Let $\omega$ be a weight. If $E \subset \mathbb{R}^{n}$ for $n \geq 2$, we define the weighted, $p$-variational capacity $\operatorname{cap}_{\omega}^{p}(E)$ by (see [7, p. 117])

$$
\begin{array}{r}
\operatorname{cap}_{\omega}^{p}(E)=\inf \left\{\int_{\mathbb{R}^{n}}|\nabla \varphi|^{p} \omega d x: \varphi \in C_{0}^{\infty}\left(\mathbb{R}^{n}\right), 0 \leq \varphi \leq 1,\right. \\
\left.E \subset \operatorname{Int}\left(\left\{x \in \mathbb{R}^{n}: \varphi(x)=1\right\}\right)\right\},
\end{array}
$$

where $\operatorname{Int}(E)$ stands for the interior of a set $E \subset \mathbb{R}^{n} ; c_{\omega}^{p}(\mu ; t)$ denotes the $p$ variational capacity minimizing function of $t \in(0, \infty)$ associated with a nonnegative measure $\mu$ on $\mathbb{R}_{+}^{n+1}$ :

$$
c_{\omega}^{p}(\mu ; t)=\inf \left\{\operatorname{cap}_{\omega}^{p}: \text { bounded open } O \subset \mathbb{R}^{n}, \mu(T(O))>t\right\},
$$

where

$$
\begin{aligned}
& T(O)=\left\{(t, x) \in \mathbb{R}_{+}^{n+1}: B(x, t) \subset O\right\}, \\
& \mathbb{R}_{+}^{n+1}=\mathbb{R}^{n+1} \bigcap\left\{x_{n+1}>0\right\} .
\end{aligned}
$$

Next, we give the Carleson embeddings for weighted Sobolev spaces. 
Theorem 3. Let $1 \leq q<\infty, 1 \leq p<n, \omega \in A_{1}\left(\mathbb{R}^{n}\right)$ and $\mu$ be a non-negative measure on $\mathbb{R}_{+}^{n+1}$. Then the following four conditions are equivalent:

(a) $\left(\int_{\mathbb{R}_{+}^{n+1}}\left|e^{t^{2} \Delta} f(x)\right|^{q} d \mu(x, t)\right)^{1 / q} \leq C\|\nabla f\|_{L^{p}(\omega)}, \forall f \in \dot{W}_{\omega}^{1, p}\left(\mathbb{R}^{n}\right)$;

(b) $\sup _{\lambda>0} \lambda\left(\mu\left(\left\{(t, x) \in \mathbb{R}_{+}^{n+1}:\left|e^{t^{2} \Delta} f(x)\right|>\lambda\right\}\right)\right)^{1 / q} \leq C\|\nabla f\|_{L^{p}(\omega)}$, $\forall f \in \dot{W}_{\omega}^{1, p}\left(\mathbb{R}^{n}\right) ;$

(c) $\sup _{t>0} \frac{t^{p / q}}{c_{\omega}^{p}(\mu ; t)}<\infty$;

(d) $\sup \left\{\frac{(\mu(T(O)))^{p / q}}{\operatorname{cap}_{\omega}^{p}(O)}\right.$ : bounded open $\left.O \subset \mathbb{R}^{n}\right\}<\infty$.

Here the weighted homogeneous Sobolev space $\dot{W}_{\omega}^{1, p}\left(\mathbb{R}^{n}\right)$ (where $p \geq 1$ ) is the completion of $f$ 's in $C_{0}^{\infty}\left(\mathbb{R}^{n}\right)$ (all infinitely differentiable functions with compact support in $\mathbb{R}^{n}$ ) with respect to the norm $\|f\|_{\dot{W}_{\omega}^{1, p}\left(\mathbb{R}^{n}\right)}=\|\nabla f\|_{L_{\omega}^{p}\left(\mathbb{R}^{n}\right)}<\infty$, and the heat kernel on $\mathbb{R}^{n} \times \mathbb{R}^{n}$ is defined by

$$
e^{t \Delta}(x, y)=(4 \pi t)^{-\frac{n}{2}} \exp \left(-\frac{|x-y|^{2}}{4 t}\right), \quad t \in(0, \infty),(x, y) \in \mathbb{R}^{n} \times \mathbb{R}^{n} .
$$

We remark that Theorem 3 extends Theorem 1.2 in [8].

To prove Theorem 3 , we need the following results.

Lemma 1. Let $\omega \in A_{1}\left(\mathbb{R}^{n}\right)$ and $1 \leq p<\infty$. Then

$$
\int_{0}^{\infty} \operatorname{cap}_{\omega}^{p}\left(\left\{x \in \mathbb{R}^{n}: M f(x)>\lambda\right\}\right) d \lambda^{p} \leq C\|\nabla f\|_{L^{p}(\omega)}, \forall f \in \dot{W}_{\omega}^{1, p}\left(\mathbb{R}^{n}\right) .
$$

Proof. We first prove the following inequality:

$$
\int_{0}^{\infty} \operatorname{cap}_{\omega}^{p}\left(\left\{x \in \mathbb{R}^{n}:|f(x)|>\lambda\right\}\right) d \lambda^{p} \leq C\|\nabla f\|_{L^{p}(\omega)}, \forall f \in \dot{W}_{\omega}^{1, p}\left(\mathbb{R}^{n}\right) .
$$

Indeed, by the monotonicity of capacity, the integral on the left-hand side in (5) does not exceed

$$
I:=\sum_{j=-\infty}^{\infty} 2^{j p} \operatorname{cap}_{\omega}^{p}\left(\left\{x:|f(x)|>2^{j}\right\}\right) .
$$

Let $\lambda_{\epsilon} \in C^{\infty}(\mathbb{R}), 0 \leq \lambda_{\epsilon} \leq 1, \lambda_{\epsilon}(t)=1$ for $t \geq 1, \lambda_{\epsilon}(t)=0$ for $t \leq 0$, $0 \leq \lambda_{\epsilon}^{\prime}(t) \leq 1+\epsilon, \epsilon>0$ and let

$$
f_{j}(x)=\lambda_{\epsilon}\left(2^{1-j}|f(x)|-1\right) .
$$


Without loss of generality, we can assume that $f \in C_{0}^{\infty}\left(\mathbb{R}^{n}\right)$. Obviously, one has $f_{j} \in C_{0}^{\infty}\left(\mathbb{R}^{n}\right), 0 \leq f_{j} \leq 1$ and

$$
E_{j}:=\left\{x:|f(x)|>2^{j}\right\} \subset \operatorname{Int}\left(\left\{x \in \mathbb{R}^{n}: f_{j}(x)=1\right\}\right),
$$

by the definition of $\operatorname{cap}_{\omega}^{p}\left(E_{j}\right)$, we then have

$$
\begin{aligned}
I & \leq C \sum_{j=-\infty}^{\infty} 2^{j p} \int_{\mathbb{R}^{n}}\left|\nabla f_{j}(x)\right|^{p} \omega(x) d x \\
& =C \sum_{j=-\infty}^{\infty} 2^{j p} \int_{\left\{x \in \mathbb{R}^{n}: 2^{j-1} \leq|f(x)|<2^{j}\right\}}\left|\nabla f_{j}(x)\right|^{p} \omega(x) d x \\
& \leq C \sum_{j=-\infty}^{\infty} \int_{\left\{x \in \mathbb{R}^{n}: 2^{j-1} \leq|f(x)|<2^{j}\right\}}\left[\lambda_{\epsilon}^{\prime}\left(2^{1-j}|f(x)|-1\right)\right]^{p}|\nabla f(x)|^{p} \omega(x) d x \\
& \leq C(1+\epsilon)^{p} \int|\nabla f(x)|^{p} \omega(x) d x .
\end{aligned}
$$

Letting $\epsilon$ tend to zero, we obtain (5).

Let us proceed to the proof. We consider two cases for $p$.

Case 1, $p=1$. From (3.5.4) in [7, p. 117] we know that $\operatorname{cap}_{\omega}^{1}(E) \simeq H_{\omega}^{n-1}(E)$. This relation, together with (5) and Theorem 1 with respect to the $(n-1)$-dimensional Hausdorff capacity yields

$$
\int_{0}^{\infty} \operatorname{cap}_{\omega}^{1}\left(\left\{x \in \mathbb{R}^{n}: M f(x)>\lambda\right\}\right) d \lambda \leq C\|\nabla f\|_{L^{1}(\omega)}, \forall f \in \dot{W}_{\omega}^{1,1}\left(\mathbb{R}^{n}\right) .
$$

Case 2, $1<p<\infty$. By the boundedness of $M$ on $L^{p}(\omega)$ and applying the same argument as in the proof of Theorem 1.4 in [4], we know that for $f \in$ $C_{0}^{\infty}\left(\mathbb{R}^{n}\right)$

$$
|\nabla(M f)(x)| \leq M(|\nabla f|)(x), \quad \text { a.e. } x \in \mathbb{R}^{n} .
$$

Hence

$$
\||\nabla(M f)|\|_{L^{p}(\omega)} \leq C\|M(|\nabla f|)\|_{L^{p}(\omega)} \leq C\|\nabla f\|_{L^{p}(\omega)} .
$$

From this and (5), we can obtain the desired result. 
Lemma 2. Let $\omega \in A_{1}\left(\mathbb{R}^{n}\right)$ and $1 \leq p<\infty$. Given $f \in \dot{W}_{\omega}^{1, p}\left(\mathbb{R}^{n}\right), \lambda>0$ and a non-negative measure $\mu$ on $\mathbb{R}_{+}^{n+1}$, let

$$
E_{\lambda}(f)=\left\{(t, y) \in \mathbb{R}_{+}^{n+1}:\left|e^{t^{2} \Delta} f(y)\right|>\lambda\right\}
$$

and

$$
O_{\lambda}(f)=\left\{x \in \mathbb{R}^{n}: \sup _{|x-y|<t}\left|e^{t^{2} \Delta} f(y)\right|>\lambda\right\}
$$

Then the following four statements are true:

(a) For any natural number $k$,

$$
\mu\left(E_{\lambda}(f) \cap T(B(0, k))\right) \leq \mu\left(T\left(O_{\lambda}(f) \cap B(0, k)\right)\right) .
$$

(b) For any natural number $k$,

$$
\operatorname{cap}_{\omega}^{p}\left(O_{\lambda}(f) \cap B(0, k)\right) \geq c_{\omega}^{p}\left(\mu ; \mu\left(T\left(O_{\lambda}(f) \cap B(0, k)\right)\right)\right) .
$$

(c) There exists a dimensional constant $\theta_{1}>0$ such that

$$
\sup _{|x-y|<t}\left|e^{t^{2} \Delta} f(y)\right| \leq \theta_{1} M f(x), \quad x \in \mathbb{R}^{n} .
$$

(d) There exists a dimensional constant $\theta_{2}>0$ such that

$$
(t, x) \in T(O) \Rightarrow e^{t^{2} \Delta}|f|(x) \geq \theta_{2},
$$

when $O$ is a bounded open set contained in $\operatorname{Int}\left(\left\{x \in \mathbb{R}^{n}: f(x)=1\right\}\right)$.

The proof of Lemma 2 can be found in [8, p. 285].

Proof of Theorem 3. Adapting the same arguments as in [8, pp. 290-291], and using Lemmas 1 and 2, we can obtain the desired result.

Acknowledgments. The author would like to thank the referee for some very valuable suggestions. 


\section{Bibliography}

[1] D. R. Adams, A note on Choquet integrals with respect to Hausdorff capacity, in: Function spaces and applications (Lund, 1986), pp. 115-124, Lecture Notes in Math. 1302, Springer, Berlin, 1988.

[2] D. R. Adams, Choquet integrals in potential theory, Publ. Mat. 42 (1998), no. 1, 3-66.

[3] J. García-Cuerva and J. L. Rubio de Francia, Weighted Norm Inequalities and Related Topics, North-Holland Mathematics Studies 116, Notas de Matematica [Mathematical Notes] 104, North-Holland Publishing Co., Amsterdam, 1985.

[4] J. Kinnunen, The Hardy-Littlewood maximal function of a Sobolev function, Israel J. Math. 100 (1997), 117-124.

[5] V. G. Maz'ya, G. Sobolev Spaces, Springer Series in Soviet Mathematics, SpringerVerlag, Berlin, 1985 (translated from the Russian).

[6] J. Orobitg and J. Verdera, Choquet integrals, Hausdorff content and the HardyLittlewood maximal operator, Bull. London Math. Soc. 30 (1998), no. 2, 145-150.

[7] B. O. Turesson, Nonlinear Potential Theory and Weighted Sobolev Spaces, Lecture Notes in Mathematics, 1736. Springer-Verlag, Berlin, 2000.

[8] J. Xiao, Carleson embeddings for Sobolev spaces via heat equation, J. Differential Equations 224 (2006), no. 2, 277-295.

Received June 19, 2009; revised June 8, 2010.

\section{Author information}

Lin Tang, LMAM, School of Mathematical Science, Peking University, Beijing 100871, P. R. China.

E-mail: tanglin@math.pku.edu.cn 